\title{
COOPERATIVAS AGROALIMENTARIAS E IMPACTO DE SU ESTRATEGIA EN EL DESARROLLO RURAL: ANÁLISIS CUALITATIVO EN CASTILLA-LA MANCHA
}

\author{
POR \\ Ignacio RUIZ GUERRA ${ }^{1}$ y \\ Víctor Manuel MARTÍN LÓPEZ²
}

\section{RESUMEN}

Ante la situación que vivimos con una duración no previsible, y unas consecuencias no cuantificables en estos momentos, debemos atender a la caracterización y conocimiento profundo y pormenorizado del sector para poder ayudar en la toma de decisiones de las cooperativas, teniendo en cuenta las necesidades de profesionalización que tienen que acometer. Es por ello que los autores siguen afirmando que el sector cooperativo necesita de una reestructuración que conlleve una profesionalización de sus cuadros de mando.

La realización de este análisis DAFO ayuda a la determinación de las debilidades y las oportunidades que surgen en los momentos de crisis. Si la concepción que tenemos del término crisis es negativa, en otras culturas tiene una connotación perfectamente entendible como positiva por ser la apertura a nuevas oportunidades.

El desarrollo rural de los municipios en los que están implantadas estas cooperativas agroalimentarias tiene mucho que ver con las decisiones que tomen los cuadros de mando que las dirigen.

Toda vez que se realiza este tipo de análisis, los cuadros de mando van a tener una mayor disposición de información para llevar a cabo su labor de toma de decisiones, lo que

\footnotetext{
${ }^{1}$ Doctor en Ciencias Económicas por la Universidad de Granada. Jefe de Estudios, Análisis y Prospectiva de la Fundación Caja Rural de Castilla-La Mancha, nachoruizguerra@gmail.com .

${ }^{2}$ Doctor en Ciencias Empresariales por la Universidad de Castilla-La Mancha. Director General de Caja Rural de Castilla-La Mancha, iruiz@cajaruralclm.es .
}

REVESCO No 111 - Segundo Cuatrimestre 2013 - ISSN: 1885-8031 - www.ucm.es/info/revesco

http://dx.doi.org/10.5209/rev_REVE.2013.v111.426750

Fecha de recepción: 12/09/2011

Fecha de aceptación: 05/04/2013 
conllevará una mejor situación a la hora de encarar las dificultades que ya acontecen en los mercados.

Palabras clave: Mercados, envejecimiento, renta, oportunidades.

Claves EconLit: O180, Q130, R230, L100.

\title{
AGRI-FOOD COOPERATIVES AND STRATEGIC IMPROVE IN A RURAL DEVELOPMENT: QUALITATIVE ANALYSIS IN CASTILLA-LA MANCHA
}

\begin{abstract}
Given the situation we live with a duration unpredictable and unquantifiable consequences at the moment, we must look to the characterization and depth of insight and industry to assist in decision-making cooperatives, taking into account the needs of professionalism they have to undertake. After many years of insisting on this point, the authors continue to argue that the cooperative sector, requires a restructuring that will lead to a professionalization of its dashboards.
\end{abstract}

The realization of this SWOT analysis helps to determine the weaknesses and the opportunities that arise in times of crisis. If the conception we have of the word crisis is negative, in other cultures has a connotation perfectly understandable as positive for being open to new opportunities.

Rural development in the municipalities in which they operate these agribusiness cooperatives has much to do with the decisions taken by the dashboards that run them.

Every time you perform this type of analysis, scorecards will have a greater availability of information to carry out their work of making decisions, which will result in a better position when facing the difficulties which occur in markets.

Keywords: Markets, age, income, opportunities.

\section{INTRODUCCIÓN}

A finales de la década de 1960, un grupo de profesores del Harvard Business School liderados por Kenneth R. Andrews y C. Roland Christensen desarrollaron una sencilla 
herramienta de análisis empresarial que denominaron SWOT (acrónimo de Strengths, Weaknesses, Opportunities, Threats; Learned et al., 1969; Andrews, 1971), y cuya traducción habitual al español es DAFO (Fortalezas, Oportunidades, Debilidades, Amenazas).

Desde un primer momento, y a lo largo de los años, el análisis DAFO se ha mostrado muy eficaz para el control de variables en la gestión empresarial hasta convertirse, hoy en día, en una técnica imprescindible para el diagnóstico de la situación actual de una empresa (Bradford et al., 1999; Santos, 2001). El análisis DAFO ha alcanzado, así mismo, gran importancia dentro de la dirección estratégica de la empresa (Hindle y Lawrence, 1993).

Por otra parte, la industria agroalimentaria en Castilla-La Mancha supone, en muchos casos de manera importante, una dependencia directa de las ayudas económicas europeas, esto es, desde el punto de vista de la economía regional, las cooperativas que surgieron hace casi 40 ó 50 años, como medio de protección y proyección de los agricultores, tienen un perfil financiero de riesgo dada su alta dependencia de las subvenciones para el mantenimiento de los niveles de renta similares a los del medio urbano. Es una de las ramas más importantes del tejido manufacturero español (Juste, 2002).

El pilar principal de la industria agroalimentaria de Castilla-La Mancha está apoyado en el sector cooperativo de manera muy importante, y dado que, desde el punto de vista industrial, las cooperativas suponen la única industria de envergadura en algunos municipios, y teniendo en cuenta la atomización municipal que caracteriza a la región castellano manchega, quiere decir que, si en los más de 900 municipios con los que cuenta, hay una cooperativa, quiere decir que existe, según la media, un número aproximado de 400 familias asociadas a la cooperativa y que trabajan en pro de la mejora económica de su municipio y sus familias a través de la unión con el resto de los vecinos (CEPES, 2010).

La población de los municipios de esta región se caracteriza por ser bastante baja, hasta el punto que, el 90\% de la población de Castilla-La Mancha reside en municipios de menos de 15.000 habitantes, una estadística a tener en cuenta, e incluso, más llamativo es que existen ciertas zonas de la geografía regional que tiene unas densidades de población inferiores a zonas desérticas de Siberia (zona de la Alcarria de Guadalajara), lo que porcentualmente otorga una importancia mayor a la información anterior sobre familias asociadas a las cooperativas. 
Actualmente nos encontramos en una situación que se caracteriza por los continuos cambios y transformaciones, situaciones y contextos nuevos, con nuevas preguntas que exigen nuevas respuestas, nuevos escenarios que unidos a fracturas sociales, nuevas exigencias y concentraciones económicas provocan como reacción la fortaleza de los valores de la solidaridad (Salinas Ramos, Herranz de la Casa, 2006).

Tradicionalmente, las áreas rurales y menos industrializadas han sido las responsables de satisfacer de productos agrícolas a las zonas más desarrolladas, además recientemente se le han unido las responsabilidades de preservar el medioambiente (Mozas, Bernal, 2006). Los especiales hechos de la actualidad, no pasan desapercibidos para el medio rural, que también sufre esas transformaciones, lo que obliga a construir nuevos diálogos y relaciones entre los diferentes agentes económicos involucrados en los procesos de desarrollo rural.

Se trata de hacer frente a un conjunto de desafíos esencialmente sociales y no exclusivamente económicos, porque el desarrollo territorial es un proceso social y multidimensional que debe partir de condiciones concretas de cada territorio rural, hacia un conjunto de sociedades territorializadas que buscan simultáneamente la eficiencia, la calidad, la flexibilidad, la agilidad y la sinergia en torno a redes de colaboración recíproca (Salinas Ramos, Herranz de la Casa, 2006).

En este contexto es necesario el fortalecimiento empresarial de las organizaciones de pequeños y medianos productores, donde la Economía Social juega un papel importante. La Economía Social no sólo afronta la globalización desde la sostenibilidad con un fuerte compromiso con lo local, con el desarrollo rural, precisamente para frenar la deslocalización de la población, sino que, generando empleo crea cohesión social y riqueza. En definitiva, contribuye a la construcción de una sociedad más plural y más democrática, se capitalizan los recursos propios de la tierra, tanto los materiales como los personales (Salinas Ramos, Herranz de la Casa, 2006)

Entre las opciones que habitualmente se han barajado para llevar a cabo las actuaciones de desarrollo económico está la potenciación del cooperativismo. El cooperativismo agrario y las organizaciones de Economía Social son las abanderadas del desarrollo rural, que tienen como principal oponente a la cada vez más amplia brecha digital. El binomio cooperativas y nuevas tecnologías tiene un gran potencial de cooperación para 
impulsar el desarrollo rural y sostenible, pero también éste presenta grandes dificultades para que el conjunto pueda prosperar (Juliá Igual, García Martínez, Meliá Martí, 2006).

El término "desarrollo rural" lleva de la mano otros conceptos como "desarrollo sostenible" o "sostenibilidad ética", que suponen el crecimiento económico sostenido y duradero en el tiempo de una región, pero basado en una gestión ética y responsable para con el medio ambiente y la sociedad en general.

En la búsqueda de una definición próxima al desarrollo rural, utilizando la expuesta por autores como Francisco Salinas Ramos, José $\mathrm{M}^{\mathrm{a}}$ Herranz de la Casa o $\mathrm{M}^{\mathrm{a}}$ de los Ángeles Nogales Naharro, podemos describirlo como: un proceso de revitalización equilibrado y autosostenible del mundo rural, basado en la potenciación de recursos económicos, sociales y medioambientales mediante una política regional, concebido más como un conjunto de acciones y de actividades que una búsqueda de resultados.

Este estudio pretende constituir una herramienta para la reflexión en el marco de la dirección estratégica en el sector cooperativo en el que debe desenvolverse para desarrollar al máximo sus capacidades y ventajas competitivas sobre la competencia. El estudio se fundamenta en la recopilación de información sobre el sector a partir de la consulta de bibliografía especializada, la consulta de cooperativas y una serie de entrevistas con expertos del sector cooperativo (Universidad de Castilla-La Mancha, Administración regional, Caja Rural de Castilla-La Mancha, etc.).

Cuando ha sido posible, se ha realizado un análisis cuantitativo de las informaciones (distribución estadística de las respuestas, análisis de balances o la creación de modelos de estrategias de producción, etc). En otros casos, solo fue posible un tratamiento cualitativo de las entrevistas y así se ha plasmado en el análisis DAFO llevado a cabo durante la realización del presente trabajo.

\section{LA METODOLOGÍA DAFO EN EL ÁMBITO EMPRESARIAL}

El objetivo fundamental del análisis es sintetizar en una matriz resumen con cuatro campos principales, la evaluación de las fortalezas, debilidades, de la propia empresa, junto con las amenazas y oportunidades que provienen del exterior. Se pretende perfilar una estrategia empresarial adecuada para lograr un óptimo ajuste entre su capacidad interna y su posición competitiva en el entorno empresarial. Para ello, la empresa ha de decidir cuáles son 
las variables apropiadas a utilizar según los mercados y segmentos en los que compite. (Pearce, 1982; Ventura, 1994; Hax y Majluf, 1996).

El producto del análisis DAFO debería ser la definición, o cuando menos la orientación, el sesgo de las estrategias o cursos de acción y actuación que la organización debería adoptar y perseguir en función de las relaciones que se establezcan entre los cuadrantes de la Matriz SWOT (DAFO en inglés). No en vano en la adopción de la estrategia definitiva pueden incorporarse nuevos elementos o criterios que perfeccionen $o$ complementen los resultados del DAFO.

Los objetivos principales en el uso del método DAFO son dos:

- Detectar problemas y diagnosticar situaciones;

- Buscar soluciones y programar acciones.

\section{Estrategias Defensivas:}

- Cuando las Fortalezas son superiores a las Amenazas: La organización está preparada para enfrentarse a las amenazas porque dispone de numerosas y bien consolidadas fortalezas. La organización puede afrontar nuevos desafíos enfrentándose a los riesgos, el signo del combate será positivo y el resultado será la potenciación de las fortalezas de la organización.

- Cuando las Amenazas son superiores a las Fortalezas: La organización con sus actuales características derivadas de sus factores internos no puede abordar nuevas singladuras, por lo que las amenazas se traducen a medio y corto plazo en debilidades. 


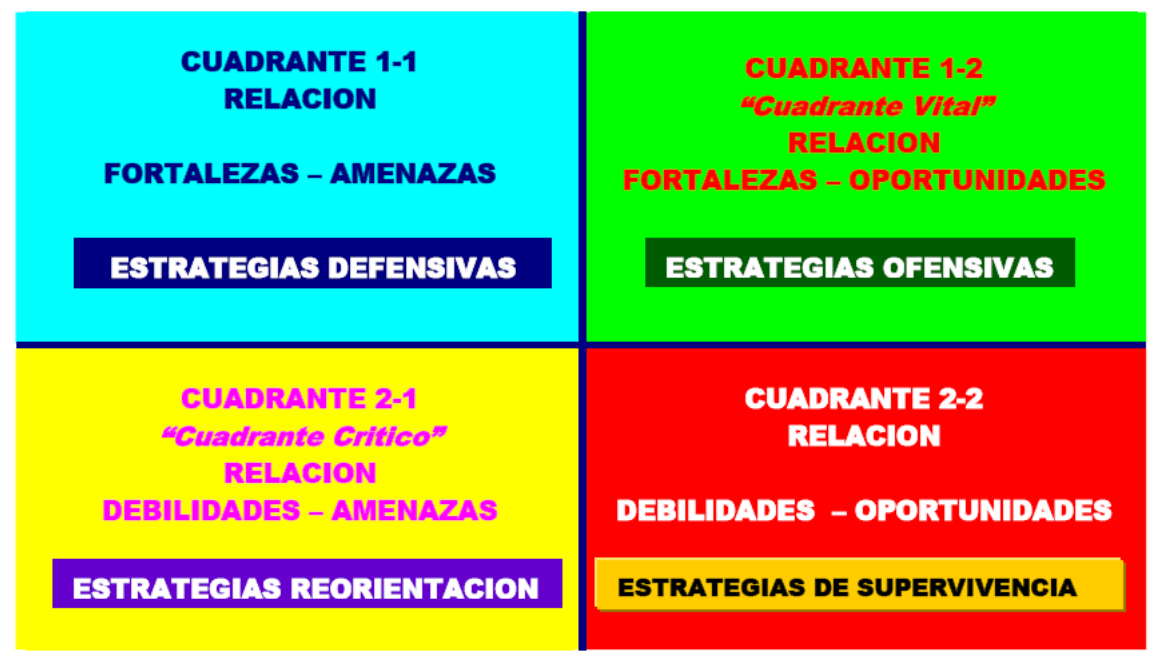

Fuente: Elaboración propia a partir de Andrews y Christensen, 1980.

\section{Estrategias Ofensivas:}

- Las Oportunidades estimulan y potencian las Fortalezas: Las oportunidades existentes generan un contexto de actuación positivo que galvaniza las fortalezas existentes y la solidez de las mismas permiten aprovechar al máximo las oportunidades. La organización podría adoptar estrategias de crecimiento.

Estrategias de Supervivencia:

- Las Amenazas potencian y aumentan las Debilidades: Las debilidades y carencias de la organización son de tal índole que no permiten a la organización afrontar las amenazas externas. La organización puede estar en peligro a corto o medio plazo.

\section{Estrategias de Reorientación:}

- La organización no puede aprovechar las buenas oportunidades existentes por sus acentuadas Debilidades: Las carencias de la organización impiden que esta pueda aprovechar el contexto favorable que plantean las oportunidades existentes.

- Las oportunidades existentes son de tal calibre que actúan como estímulo y revulsivo para la organización: Por ello la organización establece un programa de acciones específicas para la superación de sus debilidades y reorientar sus estrategias anteriores. 
Durante la realización del presente trabajo, en la fase de diagnóstico procedimos a la síntesis de la información recopilada en la fase de documentación (sobre las publicaciones existentes y relacionadas), y la posterior fase de captación de datos primarios y secundarios, para posteriormente procesar el cúmulo de información procedente del análisis de situación de manera práctica (Andrews y Christensen, 1980).

Siguiendo los tres tipos de clasificaciones de las estrategias genéricas que han quedado plasmadas en la literatura académica como un referente para todos los que abordan estos temas, nos dispusimos a plantear, a partir de Ansoff (1965) y su derivación de la matriz, la agrupación de las estrategias en cuatro grupos:

1. Penetración del mercado: se basa en los productos y mercados existentes de antemano e intenta la expansión en el mercado directamente o a través de la adquisición de empresas ya existentes.

2. Desarrollo del producto: busca la ampliación del producto o la venta de uno nuevo en un mercado ya existente.

3. Desarrollo del mercado: intenta ampliar el negocio buscando nuevos segmentos de mercado o nuevos canales de distribución, aunque también pueden buscar la consolidación del mercado al retirarse de aquellos segmentos que ya no se consideran eficientes para la empresa.

4. Expansión geográfica o diversificación: busca ofrecer nuevos productos en nuevas áreas geográficas, es decir, investigar sobre nuevos productos que se dirijan a clientes que no se tiene en este momento.

En cuanto a la metodología cualitativa llevada a cabo, se ha realizado un análisis cualitativo de entrevistas personales a responsables y miembros de consejos rectores de las cooperativas, para que, de manera anónima, aparecieran en el trabajo las diferentes visiones que desde dentro existen en el sector cooperativo. Del mismo modo que se ha procesado esa información para la realización del Análisis DAFO, se ha llevado a cabo un desarrollo de conclusiones de la realidad del sector cooperativo agroalimentario de Castilla-La Mancha. 


\section{SECTOR COOPERATIVO EN CASTILLA-LA MANCHA: ALMAZARAS Y BODEGAS}

El tamaño de las almazaras es pequeño, lo que va a dificultar llevar a cabo grandes inversiones, y también conlleva el no poder estar presente en mercados y canales donde la competencia comercial es alta, y existen grandes grupos que dominan importantes cuotas de mercado. Dos indicadores se han tenido en cuenta en esta investigación para medir el tamaño de las cooperativas:

- Número de socios: 475 socios (CEPES, 2010).

- Número de trabajadores: el 81,59\% tienen menos de tres trabajadores fijos (sobre la media de los últimos cinco años).

El cooperativismo en España consta, a fecha 2011, con 22.595 cooperativas de las que casi 4.000 son cooperativas dedicadas a actividad agroalimentaria, dando a conocer el impacto que este modelo empresarial tiene en el denominado Tercer Sector, ya que la Economía Social en nuestro país está formada por 45.093 entidades, con 2.379 .994 empleos y 12.150.549 asociados. Al sector cooperativo le corresponden, según datos oficiales, 298.514 empleos directos (CEPES, 2011) de los que, un 30\% pertenecen a dicho sector agroalimentario. En Castilla-La Mancha de las cooperativas registradas, el porcentaje de participación más alto correspondería a organizaciones formadas en su gran mayoría por empresarios autónomos agrícolas, y de ellos, muchos pertenecen al sector agroalimentario característico de la región: vino y de aceite de oliva.

La importancia de las cooperativas, que queda manifestada en los datos socioeconómicos del medio rural y las políticas de incentivo que desarrolla la Unión Europa, no sólo tiene que ver con la producción, porque la cooperativa es un instrumento de arraigo laboral a la localidad, ya que, genera rentas para el agricultor, además da trabajo directo e indirecto a muchas personas, que de otra forma se verían obligadas a emigrar hacia las grandes ciudades en busca de trabajo. 
Tabla 1. Tabla evolución cooperativas constituidas en Castilla-La Mancha

\begin{tabular}{|c|c|}
\hline & $\begin{array}{c}\text { Castilla-La } \\
\text { Mancha }\end{array}$ \\
\hline $\mathbf{2 0 0 7}$ & 11 \\
\hline $\mathbf{2 0 0 8}$ & 5 \\
\hline $\mathbf{2 0 0 9}$ & 3 \\
\hline $\mathbf{2 0 1 0}$ & 0 \\
\hline $\mathbf{2 0 1 1}$ & 0 \\
\hline $\mathbf{2 0 1 2}$ & 2 \\
\hline TOTAL & 21 \\
\hline
\end{tabular}

Fuente: Ministerio de Empleo y Seguridad Social (2012).

Refiriéndonos a las cooperativas almazaras su negocio principal es producir aceite, aunque un $41 \%$ de las mismas ofrece otro servicio más a sus socios o se dedica a otra actividad. También existe un 9\% que tiene una actividad compartida, siendo el $90 \%$ de ella la bodega, para transformar la uva en vino y derivados.

Tabla 2. Cooperativas constituidas en España.

\begin{tabular}{|c|c|}
\hline & España \\
\hline $\mathbf{2 0 0 7}$ & 28 \\
\hline $\mathbf{2 0 0 8}$ & 48 \\
\hline $\mathbf{2 0 0 9}$ & 25 \\
\hline $\mathbf{2 0 1 0}$ & 25 \\
\hline $\mathbf{2 0 1 1}$ & 38 \\
\hline $\mathbf{2 0 1 2}$ & 3 \\
\hline TOTAL & 167 \\
\hline
\end{tabular}

Fuente: Ministerio de Empleo y Seguridad Social (2012).

El mayor número de empleo es generado por:

1. Cooperativas con $75 \%$

2. Sociedades Limitadas con un $17 \%$

3. Sociedades Anónimas con un $7 \%$ 
Tabla 3. Producción de uva y vino por hectárea.

\begin{tabular}{|l|r|r|}
\hline Años & $\begin{array}{c}\text { Producción } \\
\text { de uva } \\
\text { (.000 } \text { Tm })\end{array}$ & $\begin{array}{c}\text { Producción } \\
\text { de vino } \\
\text { (.000 hls })\end{array}$ \\
\hline $\mathbf{1 9 9 9}$ & $5.607,7$ & $33.387,7$ \\
\hline $\mathbf{2 0 0 0}$ & $6.539,8$ & $41.173,9$ \\
\hline $\mathbf{2 0 0 1}$ & $5.271,7$ & $30.950,7$ \\
\hline $\mathbf{2 0 0 2}$ & $5.934,1$ & $34.539,6$ \\
\hline $\mathbf{2 0 0 3}$ & $7.240,5$ & $42.462,4$ \\
\hline $\mathbf{2 0 0 4}$ & $7.064,2$ & $42.804,3$ \\
\hline $\mathbf{2 0 0 5}$ & $6.062,5$ & $36.436,9$ \\
\hline $\mathbf{2 0 0 6}$ & $6.595,1$ & $38.907,3$ \\
\hline $\mathbf{2 0 0 7}$ & $5.962,6$ & $35.208,7$ \\
\hline $\mathbf{2 0 0 8}$ & $5.951,6$ & $37.366,9$ \\
\hline $\mathbf{2 0 0 9}$ & $5.535,3$ & $35.489,3$ \\
\hline
\end{tabular}

Fuente: Elaboración propia a partir de datos del MAGRAMA e INE.

Tabla 4. Producción de Castilla-La Mancha de viñedo.

\begin{tabular}{|l|c|c|c|c|c|}
\cline { 2 - 6 } \multicolumn{1}{l|}{ Castilla-La Mancha } & $\mathbf{2 0 0 0}$ & $\mathbf{2 0 0 1}$ & $\mathbf{2 0 0 2}$ & $\mathbf{2 0 0 3}$ & $\mathbf{2 0 1 1}$ \\
\hline Superficie viñedo (has) & 566.429 & 572.237 & 583.585 & 566.587 & 495.494 \\
\hline $\begin{array}{l}\text { Producción total de uva } \\
\text { (Tm) }\end{array}$ & 3.340 .906 & 2.336 .625 & 2.895 .248 & 3.610 .274 & 2.734 .355 \\
\hline $\begin{array}{l}\text { Producción total de vino } \\
\text { (hls) }\end{array}$ & 21.232 .036 & 13.678 .935 & 16.081 .000 & 19.808 .000 & 35.489 .000 \\
\hline
\end{tabular}

Fuente: M.A.P.A. e I.N.E.

Tabla 5. Impacto comparativo Olivar.

\begin{tabular}{|l|r|r|r|r|}
\cline { 2 - 5 } \multicolumn{1}{c|}{} & \multicolumn{1}{c|}{ MUNDO } & \multicolumn{1}{c|}{ UE } & \multicolumn{1}{c|}{ España } & $\begin{array}{c}\text { Castilla- } \\
\text { La Mancha }\end{array}$ \\
\hline Superficie (has) & 10.700 .000 & 6.000 .000 & 2.509 .677 & 397.173 \\
\hline Número de olivos & 1.300 .000 .000 & 822.000 .000 & 282.696 .000 & 36.263 .000 \\
\hline $\begin{array}{l}\text { Producción } \\
\text { (miles de toneladas) }\end{array}$ & $2.665,5$ & 1.933 & $1.607,1$ & 63 \\
\hline $\begin{array}{l}\text { Exportaciones } \\
\text { (miles de toneladas) }\end{array}$ & 581 & 374 & 560,1 & \\
\hline $\begin{array}{l}\text { Importaciones } \\
\text { (miles de toneladas) }\end{array}$ & 568 & 96 & 40,3 & - \\
\hline
\end{tabular}

Fuente: Elaboración propia a partir de Agencia Aceite de Oliva (2012).

Cuando se anuncian las características de un determinado producto se describe lo que le hace singular: la variedad, el proceso de producción, etc., ésta va siendo una de las estrategias, no de las cooperativas, sino de las sectoriales de los productos, específicamente 
del aceite de oliva y el vino. De esta forma, la responsabilidad se divide entre las sectoriales y los responsables de producción, siendo la cooperativa la que asume un papel divulgativo de la cultura alimentaria: para diferenciar la calidad de los productos, haciendo hincapié en la importancia del producto en la dieta, etc.

El carácter innovador de las cooperativas se debe a la incorporación de las nuevas funciones relacionadas con el desarrollo líneas de negocio vinculadas a la organización de actividades culturales y de ocio. Sin detener el cumplimiento de sus funciones tradicionales, las cooperativas tienen la oportunidad de anunciar su producto y su "ser capaz de hacer", a la vez muestran el origen de la producción a aquellos que se acerquen. Ésta es una práctica de acercamiento al consumidor final y una contribución al desarrollo del territorio poniendo en valor los recursos locales.

\section{MÉTODO DAFO APLICADO A LAS COOPERATIVAS}

El trabajo de campo realizado para la presente investigación se materializó mediante entrevistas a directivos (en este caso, miembros de las juntas directivas y de los consejos rectores) y personal de cooperativas con el fin de abordar temas como la composición del Consejo Rector, la profesionalización de la gestión mediante la figura de un gerente profesional, la comercialización, las innovaciones así como la introducción de las tecnologías de la información (TIC) en el sector.

La evolución del número de cooperativas ha ido cambiando con el paso de los años y con los procesos de fusión o desaparición que se están llevando a cabo a partir de la crisis, a pesar de las dificultades por las que ha ido atravesando el sector. Hay muchas que están logrando subsistir, pero no sin dificultades, ya que, cuando no es por la situación económica globalizada, es por la paralización del consumo, que ha provocado que en los casos específicos del vino y del aceite de oliva se estén pagando precios por debajo de los costes de producción, lo que está llevando a un punto de "no retorno" a los agricultores y las cooperativas agroalimentarias, si no se producen bruscos y abundantes cambios en otra dirección. El éxito de su crecimiento y permanencia durante tantos años en el mercado ha estado en los asociados, que son grandes conocedores de su negocio, de su trabajo y de su producto, pero ahora estamos en otro momento, donde priman otros valores, e incluso ha llegado un punto en el que los valores habían desaparecido, en el ámbito empresarial, no en el cooperativo que se ha mantenido fiel a su ideario. 
Dos indicadores se han tenido en cuenta en esta investigación para medir el tamaño de las cooperativas:

- Número de socios: 475 socios (media socios 2006).

- Número de trabajadores: el 81,59 \% tienen menos de tres trabajadores fijos (sobre la media de los últimos cinco años).

Se ha comprobado la alta implicación del Consejo Rector, en especial del presidente y el secretario en las actividades directivas, llevando a cabo las acciones propuestas por el Consejo Rector, que son aprobadas por los socios democráticamente. En general no existe la figura de un gerente, pero sí la de un líder o líderes dentro del Consejo Rector, que marcan las pautas o el camino a seguir.

La edad media de los socios supera los 50 años, siendo una de las características del mundo cooperativo en general. Ya que en muchos se nota la dificultad para tener recambio generacional. La participación de las mujeres en el mundo cooperativo es muy reducida, sólo un $8 \%$ de los miembros del Consejo Rector son mujeres, y cerca del $2 \%$ el número de mujeres contratadas por el sector cooperativo.

Aunque el negocio principal son los productos agroalimentarios (vino y aceite de oliva), un $41 \%$ de las almazaras, está ofreciendo otro servicio más a sus socios o están dedicándose a otra actividad. Aunque un 9\% tiene una actividad compartida, siendo el 90\% de ella la bodega, para transformar la uva en vino y derivados.

Gráfico 2. Porcentaje de Almazaras por actividades primarias, secundarias,...

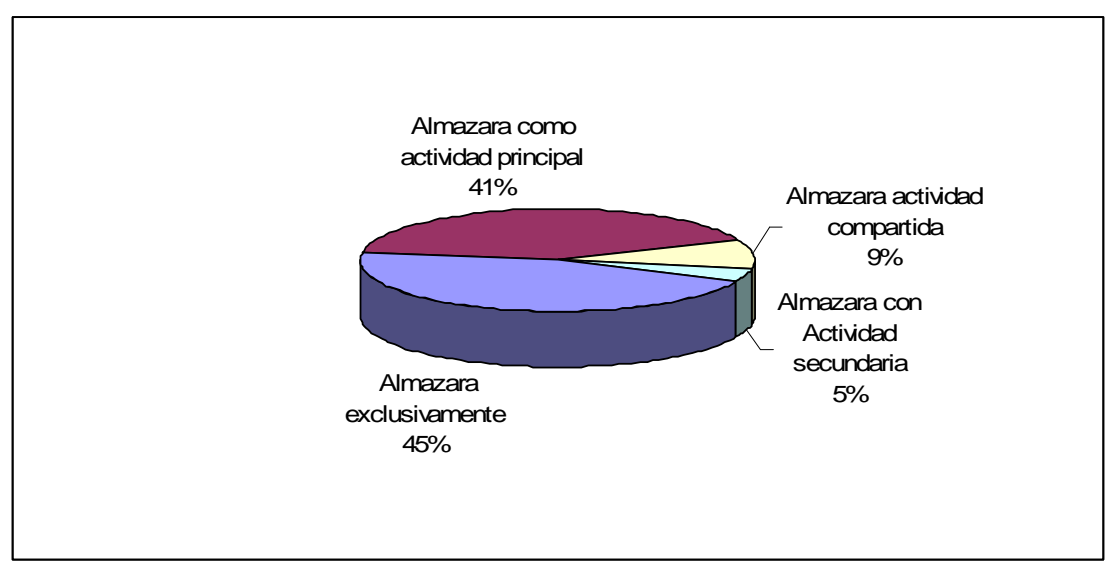

Fuente: Elaboración propia. Entrevista realizada en las Cooperativas. 
Entre los servicios accesorios o actividades secundarias de la cooperativa se encuentra como el principal el suministro de carburante. El $30 \%$ de las Cooperativas ofrecen a sus socios carburantes a un precio inferior al que se ofrece a mercado. Seguido por un $19 \%$ de venta de abonos.

La sociedad cooperativa se diferencia del resto de las sociedades mercantiles en su gestión democrática, manifestándose la misma en la participación de todos los socios en la toma de decisiones; es una sociedad de empresarios que participan en los flujos reales (participación en la producción y distribución de bienes y servicios) actuando como proveedores y/o consumidores; en los flujos financieros (de cobros y pagos, consecuencia de la traducción de los flujos reales) aportando recursos financieros; y en los flujos de información. Decisión, estableciendo democráticamente los objetivos que ha de perseguir la organización. En las características de estos flujos de información-decisión en las almazaras habrá que detenerse para tratar de explicar cómo se toman las decisiones y quién controla la gestión de la organización.

Del análisis del resultado, tanto a través del método del caso realizado y de la proposición y análisis de un modelo que recoge los principales determinantes de los resultados empresariales cooperativos, así como de la revisión teórica recogida al principio de esta tesis, se ha considerado oportuno la realización de un análisis DAFO, instrumento validado metodológicamente, y que nos permitirá realizar un análisis externo, observando aquellas amenazas y oportunidades del entorno, así como un análisis interno del sector del aceite, donde expondremos las debilidades y fortalezas de las almazaras cooperativas de la provincia de Toledo como sector concreto con génesis propia.

\subsection{Análisis externo}

\section{Amenazas}

- Dificultad para garantizar el relevo generacional en el sector agrario de Castilla-La Mancha.

- Disminución progresiva de la población activa en el medio rural y continuo envejecimiento de la media de edad de la población castellano manchega.

- Ausencia en España de una cultura de consumo prioritario de productos elaborados por cooperativas. 
- Disminución de las ayudas financieras procedentes de los fondos europeos.

- Disminución de las subvenciones por parte de las Administraciones Públicas para el sector agrario en general y del cooperativismo agrario castellanomanchego en especial.

- Mayores dificultades para el sector agroalimentario como consecuencia de los acuerdos de la Organización Mundial del Comercio (OMC).

- Las exigencias en trazabilidad, calidad y la seguridad alimentaría van a obligar a las cooperativas a segmentar a sus socios, primando a aquellos que actúen de forma acorde con los requerimientos crecientes del mercado en estos ámbitos.

- El cambio tecnológico acelerado exigirá de las cooperativas una orientación proactiva que no es frecuente en el cooperativismo agrario.

- Dificultad para el acceso a las nuevas tecnologías en el medio rural respecto del medio urbano.

- Las cooperativas tienen que operar en un mercado caracterizado por la incertidumbre y la complejidad.

- Canales comerciales no controlados por el sector cooperativo para abastecimiento en los mercados.

- El mercado de la gran distribución no podrá ser atendido, en condiciones de equilibrio de fuerzas, por las cooperativas debido a que no alcanzan una dimensión suficiente.

- Las cooperativas deberán hacer frente durante los próximos años a la tendencia de la gran distribución a gestionar marcas blancas

- La demanda está muy concentrada y la oferta, sin embargo, está atomizada.

- La desaparición paulatina de las barreras comerciales hará posible la entrada de productos de terceros países en condiciones más competitivas.

- Existencia de productos sustitutivos, de peor calidad (pero no percibida la diferencia por el consumidor), a precios más bajos. 
- Las empresas no cooperativas de la competencia dedican parte de sus esfuerzos a analizar las oportunidades que les brinda el mercado, por eso, se adaptan antes a las exigencias del mercado que las cooperativas.

\section{Oportunidades}

- La necesaria adaptación de las cooperativas a las nuevas exigencias en medioambiente, seguridad y calidad alimentaría, a la sociedad multicultural y a los nuevos hábitos de consumo, puede favorecer la diferenciación cualitativa de la marca cooperativa como valor positivo de una imagen de marca corporativa.

- Las Cooperativas pueden aprovechar su potencial como empresas de servicios agrarios para cubrir las necesidades de la creciente presencia de agricultores no profesionales.

- Las cooperativas pueden tener un importante papel en la dinamización del desarrollo rural y del territorio, dada su presencia en el medio.

- La buena imagen del cooperativismo agrario ante la sociedad puede ser un apoyo para su desarrollo comercial.

- Apoyo y asesoramiento de las entidades de crédito cooperativo. Son entidades más preocupadas por el sector cooperativo agrario, valioso instrumento para que las cooperativas puedan salir a los mercados exteriores contando con una gran experiencia y medios.

- Posibilidad de acceso a nuevos mercados exteriores debido a la ampliación de la Unión Europea y a las buenas infraestructuras de comunicación entre las distintas partes del mundo.

- Nuevos mercados y modelos para buscar nuevas líneas de desarrollo comercial: mercado de radio corto, modelo de tiendas con productos castellanomanchegos, el turismo rural.

- Posibilidad de concentrar la oferta y optimizar los medios técnicos. 
- Posibilidad de desarrollo y de obtener ventajas competitivas mediante fórmulas de intercooperación que permitan el intercambio de productos entre grupos cooperativos de otras zonas geográficas.

- Posibilidad de las cooperativas mediante procesos de integración o a través de consorcios comerciales.

- Desde el punto de vista de la Responsabilidad Social Corporativa, aspecto en el que muchas empresas están invirtiendo por diversas razones, las cooperativas por su origen fundacional, parten de una posición de ventaja, ya que su carácter es eminentemente social y están concebidas para promover el desarrollo de un colectivo o de una zona determinada

\subsection{Análisis interno}

\section{Debilidades}

- Respecto a la dirección de las cooperativas, existe una gerencia con conocimientos limitados en lo que a políticas de marketing y comercialización se refiere.

- Necesidad en las cooperativas de técnicos especialistas en productos y procesos agroalimentarios.

- Existencia de canales de comercialización en gran medida basados en la comercialización a granel.

- Escaso tamaño de las explotaciones agrarias, lo que supone una dificultad a la hora de alcanzar una determinada producción importante y sobre todo comercializadora.

- Cultura de gestión basada, casi en su totalidad, en la acumulación de experiencias de los socios.

- Falta de conocimiento del mercado globalizado, de la correcta orientación estratégica de sus productos, de las tendencias, de los gustos del consumidor, de las actuaciones de la competencia, etc. 
- Escasa realización de proyectos de Investigación, Desarrollo e innovación (I+D+i), así como escaso conocimiento de la política de ayudas para la diversificación y la innovación tecnológica.

- Pobre comunicación externa, que no consigue hacer ver al consumidor el valor añadido de los productos agroalimentarios de las cooperativas y su extraordinaria calidad.

- Escasas acciones promocionales.

- Comunicación interna entre la cooperativa y sus socios por canales clásicos, lo que conlleva a una lentitud de la toma de decisiones en inversión.

\section{Fortalezas}

- Gran potencial comercializador debido a la gran calidad de los productos agroalimentarios producidos por las cooperativas de la zona, y con herramientas suficientes para desarrollar políticas comercializadoras tanto a nivel nacional como internacional.

- Importante presencia territorial de las cooperativas, debido a su alto número de socios, lo que permite un desarrollo social y económico sostenido en las zonas en las que se asientan, aumentando la calidad de vida y dotando de estabilidad al medio rural.

- Tutela de las entidades de crédito cooperativo, en el sector cooperativo, liderando proyectos y rompiendo con la tradicional actitud de conformismo, resignación y ausencia de iniciativa.

- Estructura de costes muy controlada.

- Organización interna bien vertebrada y estabilidad de los socios en el capital social.

- Apoyo social debido a la defensa cultural y valores tales como la ecología, la calidad, la artesanía o el arraigo local, que juegan un papel importante en la defensa de los pequeños agricultores y contribuyen al desarrollo sostenible. 
- Gran volumen de producción y transformación manejado por las cooperativas, con infraestructuras modernas y sistemas de producción avanzados, contribuyendo a la estabilidad de los precios con una gama de productos cooperativos variados, de excelente calidad, saludables, de bajo coste, muy competitivos y que presentan ante los mercados una relación calidad/precio excelente.

- Aprovisionamiento de materias prima asegurado por parte de sus socios.

Una vez hemos considerado la labor desempeñada por las cooperativas respecto al empleo y el bienestar, lo que más nos ocupa es su función impulsora del desarrollo local, entendido como proceso no sólo de crecimiento, sino también de transformación socioeconómica, que lleva a cabo. Estas entidades, a través de sus múltiples formas organizativas, poseen una gran capacidad para crear un tejido empresarial allí donde se sitúan, especialmente en “... zonas rurales en despoblación donde lo que está en peligro no es sólo la posibilidad de muchas personas de vivir en su lugar de origen, sino también el abandono y degradación de espacios y de recursos de gran valor social y ecológico. Las cooperativas agro-turísticas-artesanales, el desarrollo comunitario de servicios colectivos y las asociaciones para la autoconstrucción y la generación de una oferta organizada de habitaciones y servicios de turismo rural, constituyen referentes importantes de las funciones que la economía social viene cumpliendo en áreas rurales donde existe un amplio campo de posibilidades de desarrollo" (Tomás et al., 1998).

\section{CONCLUSIONES}

En este nuevo escenario macroeconómico, social y cultural destaca el factor de la globalización, debido a la eliminación de barreras arancelarias, oscilación que hacen todavía más vulnerable a las cooperativas agrarias.

El reparto de poder y del control es una de las características de la almazara como sociedad cooperativa y que la diferencia de otras formas organizativas y cuyas funciones recaen en los socios. El poder se ejerce por todos los socios desde la asamblea general a la hora de fijar los objetivos de la organización, al igual que el control, desde el punto de vista en que los socios exigen unos resultados acordes con sus expectativas, por lo que piden explicaciones y cuentas a los miembros encargados de llevar la dirección de la almazara 
cooperativa con cierta regularidad. Los flujos de información y decisión suponen la base de la organización de la sociedad cooperativa.

En ellos está incluida la distribución de la información necesaria entre todos los miembros de la organización y el posterior proceso de toma de decisiones, en el que se fijarán las estrategias, políticas y tácticas a seguir en la organización. Estos flujos parten de la estructura organizativa de la sociedad, representada en la asamblea general de socios, la cual tiene el poder de dirección en la organización.

También es interesante para el socio la información referente a la realización de nuevas inversiones en la almazara, pues es éste, el que en última instancia decidirá en asamblea general la realización o no de dichas inversiones. La participación en la toma de decisiones se considera, pues, como un instrumento de generación de información para la dirección estratégica.

Muchas cooperativas no poseen una ventaja competitiva sostenible, pero, es bien cierto que, muchas, no la han sabido hallar por falta de un análisis de las características estructurales del sector o por no centrarse en aquellas actividades que le proporcionarían la ventaja. Sin una ventaja sólida, la almazara puede fracasar o tener un éxito limitado.

La situación de las sociedades cooperativas almazaras toledanas se caracteriza por la necesidad de una adaptación a un entorno caracterizado por:

- globalización,

- mercado abierto,

- competitividad,

- nuevos paradigmas organizacionales.

El análisis sectorial y la dirección estratégica deben permitir combatir los factores externos e internos que dificultan la supervivencia de las almazaras cuando las opiniones de algunos miembros del sector son que no existe ninguna estrategia clara y que las empresas no planifican sus estrategias.

Uno de los mayores logros de estas organizaciones es conseguir no solo un número elevado de actuaciones, sino la rapidez con las que se ejecutan, y las áreas de actuación a las 
que consigue llegar. También es cierto el fenómeno que se da en momentos de crisis económicas, en los que las dificultades son mayores para el sector empresarial en general pero para el cooperativista es completamente distinto dándose la circunstancia de que en esos mismos momentos de crisis este sector crece y obtiene mayor responsabilidad debido a que la sociedad en general reconoce el grado de compromiso con su tierra y el entorno que le rodea, por lo tanto la gente ve más que otra cosa la confianza en el sector y en los valores cooperativistas.

También se refieren al desarrollo otros factores como el aumento del nivel de vida, identificado con un incremento de la renta per cápita superior a la población; hay que considerar la calidad de los productos y no solo su cantidad, puesto que una expansión sin medida podría provocar el agotamiento de los recursos naturales de la zona; el bienestar económico se correspondería con los conceptos de cómo producimos, pero el bienestar social exige una adecuación del qué se produce.

Para una buena planificación del desarrollo rural, éste debe considerar los siguientes elementos:

- Creación de empleo local.

- Posibilidades de crecimiento económico endógeno de la zona.

- Infraestructuras y servicios públicos estratégicos.

- Instituciones regionales básicas para la buena autogestión.

- Una educación comunitaria que promueva valores e identidades regionales.

- Explotación controlada de los recursos naturales de la región.

Hay que reseñar que estos condicionantes no son iguales para todas las regiones, y que cada territorio deberá realizar una planificación del desarrollo rural en función de su geografía, historia y características singulares.

La realidad de Castilla-La Mancha está intrínsecamente ligada a la industria agroalimentaria, por su marcado carácter agrícola, y éste, a su vez, está directamente relacionado con la viabilidad del sector cooperativo, dependiente, en gran medida, de la rentabilidad que puedan obtener en la venta de los productos manufacturados. Es decir, la 
población de Castilla-La Mancha y su permanencia en sus municipios de origen dependen directamente, con mayor o menor grado, de la sostenibilidad del sector agroalimentario cooperativo, y con él, el desarrollo rural dependerá de las vías de mantenimiento y focalización innovadora de nuevas oportunidades para un futuro prometedor.

\section{BIBLIOGRAFÍA}

ANDREWS, K. R.: The concept of corporate strategy, H. Dow Jones-Irwin, Homewood, Il, 1971.

ANDREWS, K.R.: The concept of Corporate Strategy, Ed. Irwin, Homewood, Illinois, 1980.

ANSOFF, H.: La estrategia de la empresa, Ed. Universidad de Navarra, Pamplona, 1965.

HAX, A. C., MAJLUF, N. S.: The Strategic Concept and Process. A pragmatic Approach (2. ${ }^{\text {a }}$ ed.). Prentice Hall, Upper Saddle River, 1996, 440 pp.

HINDLE, T., LAWRENCE, M., Eds.: "Field Guide to Strategy - A Glossary of Essencial Tools and Concepts of Today Managers", en Harvard Business School Press, Cambridge, Massachusetts, 1993, 225 pp.

JULIÁ IGUAL, J.F., GARCÍA MARTÍNEZ, G. y MELIÁ MARTÍ, E.: Internet, cooperativismo agrario y desarrollo rural. El caso de las cooperativas de la Comunidad Valenciana. CIRIEC-España, 2006.

JUSTE CARRIÓN, J. J.: Industria agroalimentaria y desarrollo rural: algunas reflexiones sobre el caso de Castilla y León, en AA. VV. Anales de Estudios Económicos y Empresariales, $\mathrm{n}^{\circ}$ 15, 2002, pp. 189-214.

LEARNED, E., CHRISTIANSEN, C., ANDREWS, K. and GUTH, W.: Business Policy: Text and Cases, Richard D. Irwin, Homewood, Ill, 1969.

MOZAS MORAL, A. y BERNAL JURADO, E.: Desarrollo territorial y economía social. CIRIEC-España, 2006.

PEARCE, J. A.: Strategic Management: Strategy Formulation and Implementation, 1982.

RICHARD D. Irwin, Homewood, Illinois. 991 pp.

SALINAS RAMOS, F. y HERRANZ DE LA CASA, J. M.: Monográfico Economía Social y Desarrollo Rural. CIRIEC-España, 2006.

SANTOS, M.: "El análisis DAFO. Cómo valorar las debilidades, amenazas, fortalezas y oportunidades de la empresa", en Revista Emprendedores, nº 43, 2001, pp. 82-87.

VENTURA, J.: Análisis competitivo de la empresa: Un enfoque estratégico, Editorial Civitas, Madrid, 1994, 288 pp. 